\title{
First-principles calculation of chiral current and quantum self-inductance of carbon nanotubes
}

\author{
Bin Wang, ${ }^{1}$ Ruilin Chu, ${ }^{1}$ Jian Wang, ${ }^{1, *}$ and Hong $\mathrm{Guo}^{2}$ \\ ${ }^{1}$ Center of Theoretical and Computational Physics and Department of Physics, The University of Hong Kong, \\ Pokfulam Road, Hong Kong, China \\ ${ }^{2}$ Center for the Physics of Materials and Department of Physics, McGill University, Montreal, Quebec, Canada H3A 2T8
}

(Received 4 July 2009; revised manuscript received 23 November 2009; published 28 December 2009)

\begin{abstract}
We report a first-principles calculation on the current density distribution in carbon nanotubes (CNTs). Due to inversion symmetry breaking, a chiral current is found to flow in chiral CNTs. The chiral current varies continuously on each transmission plateau but jumps abruptly when the number of transmission channels is changed. A magnetic field is induced inside the CNT with a significant magnitude whose direction can be reversed by tuning the Fermi energy. Unlike a classical inductor whose inductance is a property of the geometric structure only, the self-inductance of CNT is sensitive to the Fermi energy. This quantum feature should also exist in other nanotubes.
\end{abstract}

DOI: $10.1103 /$ PhysRevB.80.235430

PACS number(s): 85.35. $-\mathrm{p}, 85.65 .+\mathrm{h}, 71.15 . \mathrm{Mb}$

\section{INTRODUCTION}

Carbon nanotubes (CNTs) have been the focus of intensive studies due to its unique geometrical structure and electronic properties. ${ }^{1,2} \mathrm{~A}$ CNT characterized by $(n, m)$ (the number of unit vectors along two directions in a graphene sheet) can be zigzag, armchair, and chiral configurations. ${ }^{3}$ Most single-wall CNTs are found experimentally to be chiral. ${ }^{4}$ The relation between $n$ and $m$ also determines whether the CNT is metallic or semiconductor. ${ }^{5}$ In addition, most of chiral CNTs with diameter less than $10 \AA$ are semiconductor because of the $\sigma-\pi$ hybridization due to the curvature of tubule wall. ${ }^{6}$

As a perfect one-dimensional conductor, the current density distribution in CNTs has attracted attention recently. ${ }^{7}$ Current density gives local information of nonequilibrium transport, thereby providing useful and vivid insight to transport properties of molecular electronics. In particular, whether or not a chiral CNT behaves as a microscopic coil has been investigated when it is metallic. ${ }^{8,9}$ Within the framework of the Boltzmann transport equation, Miyamoto et al. showed that ${ }^{10,11}$ helical current is expected for chiral $\mathrm{BC}_{2} \mathrm{~N}$ nanotube due to the anisotropic structure of $\mathrm{BC}_{2} \mathrm{~N}$ sheet. They argued that chiral CNTs do not show helical current because of the isotropic geometric structure of graphite sheet. However, helical current may be obtained by doping alkali atoms or halogen atoms in $\mathrm{CNT}^{8}$ or by mechanical stretching along the tubule axis. ${ }^{9}$ Within the framework of nonequilibrium Green's function (NEGF) method, Tsuji et $a l .{ }^{12}$ showed that a loop current can be dramatically amplified for a finite length CNT coupled with two electrodes when the energy of the injected electron is resonant with degenerate eigenstates of isolate CNT. Recently, an interesting work was carried out by Lambert $e t$ al. to investigate the chiral current of chiral CNTs. ${ }^{13}$ Based on the analytical derivation for the group-velocity components, the authors show that the chiral current of a chiral CNT can oscillate with the energy and even changes its sign. A significant magnetic field of order $0.1 \mathrm{~T}$ can be generated by a large chiral current.

To the best of our knowledge, most of the previous theoretical investigations of chiral current were based on tight- binding approximation and the electronic structure was derived from two-dimensional graphene sheet. However, the extension of $\pi$ bonds in three dimensions should not be ignored in calculating the current density distributions in CNT. In this paper, we carried out an $a b$ initio calculation within the framework of nonequilibrium Green's functions to investigate transport properties of chiral CNTs. We find that a chiral current can be generated in chiral CNT when the Fermi level is away from the Dirac point. The magnitude and direction of the chiral current are sensitive to the Fermi level. We also investigate the self-inductance of chiral CNTs that exhibits quantum nature.

\section{THEORETICAL FORMALISM AND RESULTS}

To investigate transport properties of chiral CNT, we use a state-of-the-art first-principles technique that is based on combining the density functional theory (DFT) with the Keldysh NEGFs. The basic principle and practical implementation of the NEGF-DFT formalism can be found in Ref. 14. In particular, the conventional current density $\mathbf{J}_{c}(\mathbf{r})$ is defined as ${ }^{15}$

$$
\mathbf{J}_{c}(\mathbf{r})=-\frac{e \hbar}{4 \pi m} \int \frac{d E}{2 \pi}\left[\left(\nabla-\nabla^{\prime}\right) G^{<}\left(\mathbf{r}, \mathbf{r}^{\prime}, E\right)\right]_{\mathbf{r}=\mathbf{r}^{\prime}},
$$

where $G^{<}=G^{r} \Sigma^{<} G^{a}$ is the lesser Green's function and $\Sigma^{<}$ $=i \Sigma_{\alpha} \Gamma_{\alpha} f_{\alpha}$ is the lesser self-energy. Here $\Gamma_{\alpha}$ and $f_{\alpha}$ are the linewidth function and the Fermi distribution function of a lead labeled by $\alpha$, respectively.

Before presenting numerical results, we first qualitatively discuss the physical reason why there can be a chiral current in chiral CNT using the band structure projected onto the Brillouin zone of graphite. ${ }^{1}$ Due to periodic boundary condition of the tube, the wave vector $k_{\perp}$ perpendicular to the tubule axis becomes discretized while the wave vector $k_{\|}$ parallel to the tubule axis remains continuous [see solid lines in Fig. 1 where a tight-binding model with one $\pi$ bond per atom is used for a $(5,2)$ tube]. It is known that for a CNT with $N$ hexagons per unit cell, there are $2 N$ discrete energy bands. ${ }^{1}$ Each energy band contributes to translational as well 


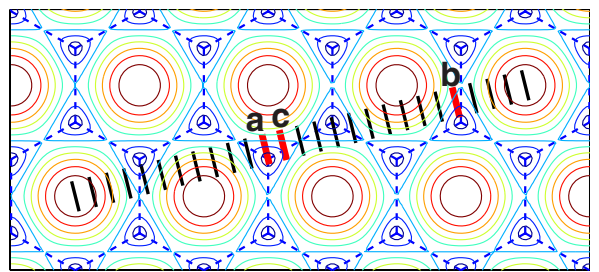

FIG. 1. (Color online) The Brillouin zone of $(5,2)$ CNT.

as cyclic current density. The current density is given by $\mathbf{J}$ $=n e \mathbf{v}$ with the group velocity $\mathbf{v}=\nabla_{k} E=\mathbf{v}_{\perp}+\mathbf{v}_{\|}$, where $\mathbf{v}_{\perp}$ $=\nabla_{k} E$. Note that the total current density is the sum of the contribution from all energy bands at the Fermi level, hence $\mathbf{v}=\sum_{i}\left(\mathbf{v}_{\perp}^{i}+\mathbf{v}_{\|}^{i}\right)$. When the Fermi energy is near the Dirac point of graphite, only one energy band labeled line a in Fig. 1 contributes to the transport. At small energies it has two intersections with the energy surface. For a chiral CNT the contribution to cyclic current density due to these two $k$ points cannot cancel completely giving rise to a positive group velocity and hence positive cyclic current. For energies on a transmission plateau, $\partial E / \partial k_{\|}^{i}$ and $\partial E / \partial k_{\perp}^{i}$ change continuously with the increase of Fermi energy. As a result, the current ratio changes continuously. When the Fermi energy increases further, additional band marked by line $b$ in Fig. 1 participates in transport with positive contribution to the cyclic current density. Therefore a jump in cyclic current density should occur at the transmission band edges. For even larger energies, a third band labeled by line c comes into play. For a $(5,2) \mathrm{CNT}$, the contribution to the cyclic current density from the third band is very large and negative-resulting a negative cyclic current density, i.e., a reversal of cyclic current may occur when additional transmission channels contribute. Although this qualitative argument is from a tight-binding model of CNT, it captures the essential physics of cyclic current which is verified by firstprinciples calculations.

In the first-principles calculation of current density, we consider a $(5,2)$ CNT whose tubule axis is set along the $z$ direction. There are 52 carbon atoms per unit cell and the geometry chiral angle is $16.1^{\circ}$. The current density consists of two parts, $J_{c}$ and $J_{z}$, i.e., cyclic current density along the circumference and translational current density along the tubule axis, respectively.

Since the conducting orbit of carbon is the $\pi$ bond while the nonconducting orbit is the $\sigma$ bond, we expect that the current density is zero on the circumference and nonzero near $\pi$ bonds. The cyclic current densities with $E_{F}$ $=1.36 \mathrm{eV}$ and $E_{F}=1.97 \mathrm{eV}$ are shown in Figs. 2(a) and 2(b), respectively. For illustration purpose, we have averaged the current density along $z$ direction by $I_{c}=\int_{0}^{z 0} J_{c} d z$ with $z 0$ as the length of unit cell. Clearly, $I_{c}$ shows different distributions at different Fermi energies. When $E_{F}<1.9 \mathrm{eV}$ there are at most five propagating channels. The cyclic currents $I_{c}$ are found to flow counterclockwise and mostly concentrated at the interior of the tube, i.e., only inner half of the $\pi$ bond carries cyclic current. When the Fermi energy is larger than $1.9 \mathrm{eV}$, two more transmission channels open up and the cyclic current becomes clockwise and distributes both inside and outside of the tube [see Fig. 2(b)]. It is interesting to note (a)

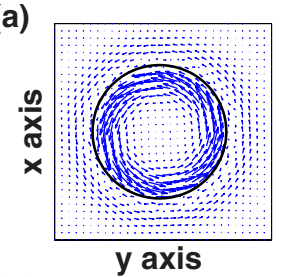

(c)

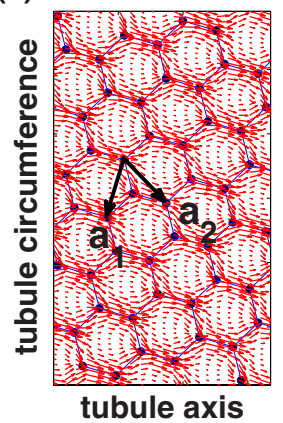

(b)

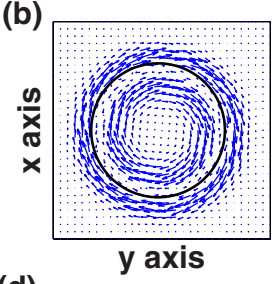

(d)

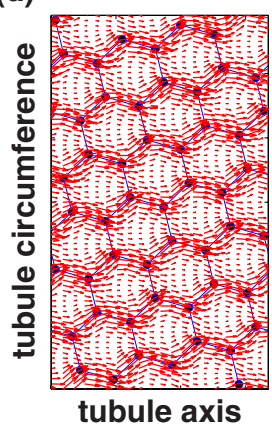

FIG. 2. (Color online) The cyclic current $I_{c}$ of $(5,2) \mathrm{CNT}$ with (a) $E_{F}=1.36 \mathrm{eV}$ and (b) $E_{F}=1.97 \mathrm{eV}$ where solid circles indicate the circumference of the CNT. (c) Projected current distribution on the unrolled $(5,2)$ CNT surface with (c) $E_{F}=1.36 \mathrm{eV}$ and (d) $E_{F}$ $=1.97 \mathrm{eV}$. The vector gives the magnitude and orientation of the current. In (c), $\mathbf{a}_{1}$ and $\mathbf{a}_{2}$ are unit vectors of hexagonal lattice.

that the distribution of translational current density $J_{z}$ carried by $\pi$ bonds is nonzero both inside and outside of the tube regardless of the Fermi energy.

In order to see more clearly how the current flows on the hexagon lattice, we use the current density projected on the tube circumference, i.e., $\mathbf{J}_{p}=\int d r \mathbf{J}(r, \theta, z)$ and unroll the CNT along the translational vector $T$ to a graphene sheet. In Figs. 2(c) and 2(d), the projected current densities are shown on one unit cell of unrolled $(5,2)$ CNT with the Fermi energies equal to 1.36 and $1.97 \mathrm{eV}$, respectively. It is clear that the current flows along $\mathrm{C}-\mathrm{C}$ bonds. For CNTs there are two possible chiral paths for the cyclic current that correspond to two sets of zigzag chains in Fig. 2(c). One of them is along $\mathbf{a}_{2}$ and the other is along $\mathbf{a}_{2}-\mathbf{a}_{1}$, where $\mathbf{a}_{1}$ and $\mathbf{a}_{2}$ are unit vectors of hexagonal lattice. For zigzag and armchair CNTs, the cyclic currents are evenly distributed on two symmetric chiral paths resulting a zero total cyclic current. For chiral CNTs, these two chiral paths are asymmetric leading to a net cyclic current. In addition, the composition of cyclic current from two chiral paths depends on energy. It is the competition between two chiral paths that determines the direction of the cyclic current. For instance, when $E_{F}=1.36 \mathrm{eV}$, a counterclockwise cyclic current occurs as shown in Fig. 2(a). With $E_{F}=1.97 \mathrm{eV}$, the current mostly traverses along the chiral path $\mathbf{a}_{2}-\mathbf{a}_{1}$ with a net clockwise cyclic current, as shown in Fig. 2(b).

Denoting $\kappa$ as the ratio of total cyclic current and total translational current, we plot $\kappa$ and transmission coefficient $T$ as a function of the Fermi energy for $(5,2)$ CNT in Fig. 3 (a). The reason that the transmission coefficient of the $(5,2)$ CNT shows a small gap around $E_{F}=0$ is due to the finite curvature of the CNT which alters the overlap of $\pi$-electron wave functions. In this energy gap, the current ratio cannot be defined since $I_{z}$ is zero. Outside of this gap the current 


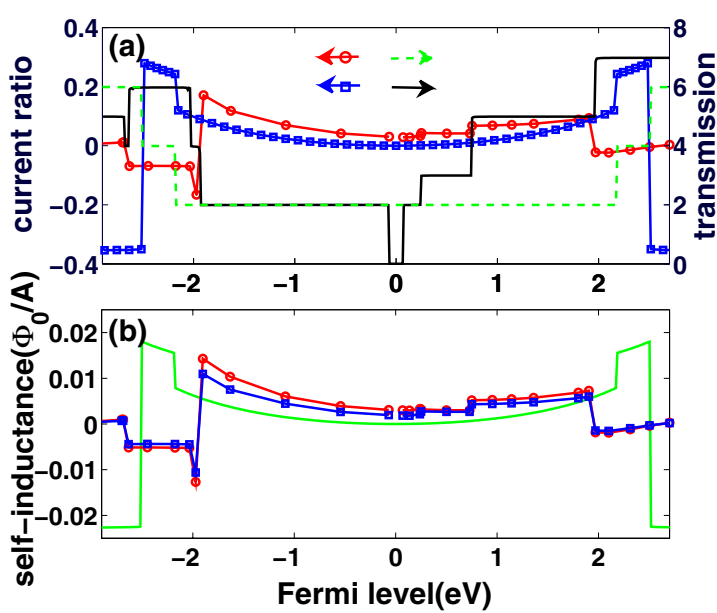

FIG. 3. (Color online) (a) Current ratio and transmission coefficient of $(5,2) \mathrm{CNT}$ as a function of Fermi level. The black line and red-circle line are from the $a b$ initio results. The green-dashed line and blue-square line are from tight-binding model. (b) The quantum inductance as a function of the Fermi level. The red-circle line and the blue-square line are calculated numerically and analytically, respectively. The green line is from tight-binding model.

ratio varies with the Fermi energy. In general, our numerical results on different CNTs show that the cyclic current is counterclockwise at small Fermi energies and clockwise at large energies. For the $(5,2)$ CNT, current ratio changes continuously on each transmission plateau except for the plateau with $T=3$. When an additional energy band contributes to the transmission, current ratio jumps and may even reverse its sign. The reversal of the current ratio corresponds to the reversal of the cyclic current. The maximum value of current ratio reaches to nearly $20 \%$ when $E_{F} \sim-1.9 \mathrm{eV}$. Intuitively, the direction of the cyclic current would be determined by the chirality of the CNT. Our result, however, shows that the direction of chiral current depends only on the Fermi energy and not the chirality. To verify that this quantum feature is generic to chiral CNTs, we have investigated current density distribution for $(8,2),(6,2)$, and $(6,4)$ CNTs and found similar behaviors. Since it is very difficult to calculate the current density for larger CNTs using ab initio methods, we have also calculated current density using a tight-binding model with one orbital per carbon atom. The tight-binding results for the $(5,2)$ CNT are shown in Fig. 3 (solid line without dots) for comparison. Since the band structures obtained from ab initio calculation and tight-binding model are different, only qualitative comparison can be made. Because calculation of cyclic current density is much easier using the tight-binding model, we have searched a number of CNTs with different chiralities and found that they all reveal the behavior of cyclic current reversal. For instance, it is found that for a $(16,4)$ CNT the cyclic current reverses its sign at $E_{F}=0.92 \mathrm{eV}$ from $\kappa=0.08$ to $\kappa=-0.06$.

A magnetic field is generated by the cyclic current in chiral CNTs which can be calculated from the vector potential $\mathbf{B}=\nabla \times \mathbf{A}$. The vector potential $\mathbf{A}$ obeys the following Poisson-like equation $\nabla^{2} \mathbf{A}(\mathbf{r})=-\mu_{0} \mathbf{J}(\mathbf{r})$, where $\mathbf{J}(\mathbf{r})$ is the current density. For example, assuming the bias voltage equal to $1 \mathrm{~V}$ and $E_{F}=1.36 \mathrm{eV}$, the calculated magnetic field (a)

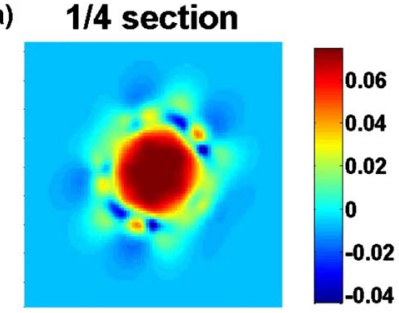

(c)

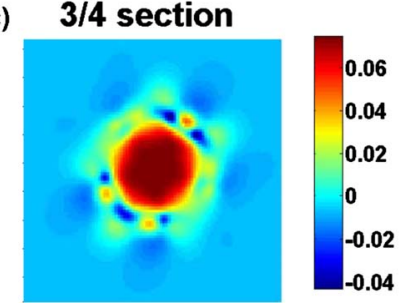

(b)

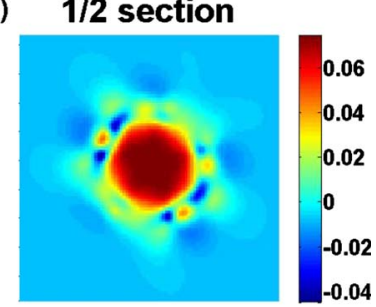

(d)

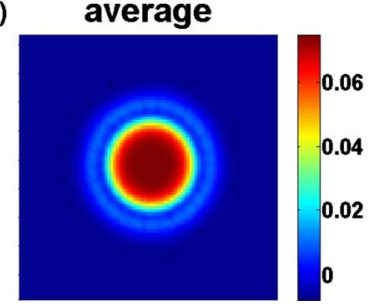

FIG. 4. (Color online) (a)-(c) The magnetic field on different cross sections of $(5,2) \mathrm{CNT}$ with $E_{F}=1.36 \mathrm{eV}$ and $V_{b}=1 \mathrm{~V}$. (d) The average magnetic field on each cross section of $(5,2)$ CNT with $E_{F}=1.36 \mathrm{eV}$ and $V_{b}=1 \mathrm{~V}$.

distribution on different cross sections of $(5,2)$ CNT is shown in Figs. 4(a)-4(c). We observe that the magnetic field lines are not completely localized inside the tube. This is because the chiral CNT is not exactly a tightly wound solenoid so that the magnetic field lines cannot be completely screened. In addition, due to the breakdown of translational symmetry for chiral CNT within the unit cell, the magnetic field is not uniform on each slice of the unit cell. However, the average magnetic field along $z$ direction in each unit cell has circular symmetry with respect to the axis of CNT as shown in Fig. 4(d). We also observe that the magnetic field is almost uniform inside the CNT. When the bias $V_{b}=1 \mathrm{~V}$ and $E_{F}=$ $-1.9 \mathrm{eV}$, the magnetic field along the axis of CNT is about $0.067 \mathrm{~T}$ (Ref. 13) and the magnetic flux $\Phi=\int B d \sigma$ is equal to $2.2 \times 10^{-6} \Phi_{0}$ with $\Phi_{0}$ as the flux quantum.

Due to the chiral flow of current, chiral CNTs behave similar to a solenoid and it is interesting to investigate the magnetic self-inductance defined as

$$
L=\frac{\Phi}{I},
$$

where $I=I_{z}$ is the current injected to the CNT. As shown in Fig. 3(b), the self-inductance versus energy shows similar behavior as the current ratio $\kappa$ in Fig. 3(a). In general, the self-inductance increases quadratically within each transmission plateau as energy increases except when incoming channels are three and six where the self-inductance is approximately a constant. Whenever an extra transmission channel is added, a jump appears in the self-inductance. Similar to the geometric capacitance, classical self-inductance depends only on the geometry of the system: the self-inductance of a classical solenoid depends only on its radius and the number of turns per unit length $N$. For chiral CNTs, our results show that self-inductance also depends on energy. This is true even at transmission plateau where $I_{z}$ is a constant. This quantum feature is an intrinsic property of chiral CNTs. 
The self-inductance of chiral CNTs can be understood semiclassically using the picture of a solenoid. In this picture, the chiral CNT consists of two solenoids, with cyclic current $I_{1}$ and $I_{2}$ along chiral paths $\mathbf{a}_{2}$ and $\mathbf{a}_{2}-\mathbf{a}_{1}$, respectively. Although the chiral path is a zigzag path, it can be approximated by a continuous path with an angle $\theta$. Using this approximation, two angles $\theta_{1}=\pi / 6+\alpha$ and $\theta_{2}=\pi / 6-\alpha$ can be defined for chiral paths $\mathbf{a}_{2}$ and $\mathbf{a}_{2}-\mathbf{a}_{1}$, respectively. Here the chiral angle $\alpha$ is the angle between the chiral path and the tubule axis and is fixed for a specific CNT. Obviously, the competition between $I_{1}$ and $I_{2}$ determines the current ratio and therefore the induced magnetic field. Using this semiclassical picture, the magnetic field can be calculated from the classical solenoid as $B=\mu_{0} n_{0}\left(I_{1} \sin \theta_{1}\right.$ $-I_{2} \sin \theta_{2}$ ). Since the net cyclic current $I_{c}$ can be determined from $I_{1}$ and $I_{2}$, the magnetic field can also be expressed as $B=\mu_{0} n_{0} I_{c}$. Therefore, the self-inductance can be calculated approximately,

$$
L=\mu_{0} n_{0} S \frac{I_{c}}{I_{z}}=\frac{\mu_{0} d_{r} a}{4 \sqrt{3} \pi} \sqrt{n^{2}+m^{2}+n m} \tan \theta,
$$

where $n$ and $m$ are the chiral indices of CNT, $S$ is the area of the CNT, $a$ is the length of the unit vector of hexagonal lattice, $d_{r}$ is the greatest common divisor of $2 m+n$ and $2 n$ $+m$, and $\tan \theta=I_{c} / I_{z}$. For $(n, m) \mathrm{CNTs}$, once $\tan \theta$ is known, the self-inductance can be determined analytically using the above formula. In Fig. 3(b), we plot the self-inductance calculated from this analytic formula (see the curve with solid square). The agreement between the semiclassical formula and the $a b$ initio data is quite good. The largest different is about $20 \%$ which is mainly due to the nonuniform distribution of magnetic field inside the CNT. With the analytic method, ${ }^{13}$ it was suggested that the optimal chiral angle is about $\pi / 10$ for which the magnetic field is the largest. Because it is difficult to obtain such an analytical expression in $a b$ initio calculation, we cannot predict which chiral angle is optimal. In Table I, numerical results from $a b$ initio calculation were listed for three CNTs where minus sign corresponds to an opposite cyclic current direction. For $(5,2)$ $\mathrm{CNT}$, the maximum value of $|\theta|$ occurs at $E_{F}=-0.19 \mathrm{eV}$ with $\theta=0.168$, magnetic field $B=0.067 \mathrm{~T}$, and selfinductance $0.014 \Phi_{0} / A$. For $(8,2) \mathrm{CNT}$, however, the largest
TABLE I. Chiral angle $\alpha$, maximum value of $\theta, B$, and $L$ for different chiral CNTs.

\begin{tabular}{lcccc}
\hline \hline & & & $B$ & \\
& $\alpha$ & $\theta$ & $(\mathrm{T})$ & $L\left(\Phi_{0} / A\right)$ \\
\hline$(5,2) \mathrm{CNT}$ & 0.281 & 0.168 & 0.067 & 0.014 \\
$(6,2) \mathrm{CNT}$ & 0.242 & 0.217 & 0.072 & 0.014 \\
$(8,2) \mathrm{CNT}$ & 0.190 & -0.273 & -0.60 & -0.06 \\
\hline \hline
\end{tabular}

$\theta=-0.273$ occurs at $E_{F}=2.17 \mathrm{eV}$ with magnetic field $B=$ $-0.6 \mathrm{~T}$ and self-inductance $-0.06 \Phi_{0} / A$. It seems that the chiral angle $\alpha$ is inversely proportional to $\theta$, but there is no obvious relationship between $\alpha$ and magnetic field (or selfinductance). In addition, we note that this magnetic field may be enhanced if the CNT is filled with magnetic atoms such as ion or cobalt.

\section{SUMMARY}

In conclusion, we found that a transport cyclic current exists for chiral CNTs that depends not only on the geometry but also its Fermi energy. As energy varies, the current ratio between cyclic current and translational current changes continuously on each transmission plateau and it jumps whenever additional transmission channel appears. Due to the cyclic current, a magnetic field of order $0.6 \mathrm{~T}$ is generated inside CNTs. The magnetic field can be used to detect the cyclic current experimentally. The quantum self-inductance is found to depend chirality index of CNTs as well as Fermi energy. We have derived a semiclassical formula for the selfinductance that is qualitatively consistent with the $a b$ initio data.

\section{ACKNOWLEDGMENTS}

This work was financially supported by RGC grant (Grant No. HKU 704308P) from the Government SAR of Hong Kong and LuXin Energy Group. H.G. is supported by NSERC of Canada, FQRNT of Québec, and Canadian Institute of Advanced Research. The authors thank X. D. Cui for useful discussions.

\footnotetext{
*jianwang@hkusub.hku.hk

${ }^{1}$ R. Saito, G. Dresselhaus, and M. S. Dresselhaus, Physical Properties of Carbon Nanotubes (Imperial College Press, London, 1998).

${ }^{2}$ S. Reich, C. Thomsen, and J. Maultzsch, Carbon Nanotubes: Basic Concepts and Physical Properties (Wiley-VCH, Berlin, 2003).

${ }^{3}$ R. Saito, M. Fujita, G. Dresselhaus, and M. S. Dresselhaus, Appl. Phys. Lett. 60, 2204 (1992).

${ }^{4}$ S. Iijima and T. Ichihashi, Nature (London) 363, 603 (1993).

${ }^{5}$ N. Hamada, S.-i. Sawada, and A. Oshiyama, Phys. Rev. Lett. 68, 1579 (1992).
}

\footnotetext{
${ }^{6}$ X. Blase, L. X. Benedict, E. L. Shirley, and S. G. Louie, Phys. Rev. Lett. 72, 1878 (1994); C. L. Kane and E. J. Mele, ibid. 78, 1932 (1997); M. Ouyang, J. Huang, C. Cheung, and C. M. Lieber, Science 292, 702 (2001).

${ }^{7}$ Y. Liu and H. Guo, Phys. Rev. B 69, 115401 (2004).

${ }^{8}$ Y. Miyamoto, A. Rubio, X. Blase, M. L. Cohen, and S. G. Louie, Phys. Rev. Lett. 74, 2993 (1995).

${ }^{9}$ Y. Miyamoto, Phys. Rev. B 54, R11149 (1996).

${ }^{10}$ Y. Miyamoto, A. Rubio, M. L. Cohen, and S. G. Louie, Phys. Rev. B 50, 4976 (1994).

${ }^{11}$ Y. Miyamoto, S. G. Louie, and M. L. Cohen, Phys. Rev. Lett.
} 
76, 2121 (1996).

${ }^{12}$ N. Tsuji, S. Takajo, and H. Aoki, Phys. Rev. B 75, 153406 (2007).

${ }^{13}$ C. J. Lambert, S. W. D. Bailey, and J. Cserti, Phys. Rev. B 78, 233405 (2008).

${ }^{14}$ J. Taylor, H. Guo, and J. Wang, Phys. Rev. B 63, 245407 (2001); 63, 121104 (2001).
${ }^{15}$ Note that this definition of $\mathbf{J}_{\mathbf{c}}(\mathbf{r})$ does not satisfy the current continuity equation in the presence of nonlocal pseudopotentials. To fix this problem, we adopt the definition of current density $\mathbf{J}(\mathbf{r})=\mathbf{J}_{\mathbf{c}}(\mathbf{r})+\mathbf{J}_{\mathbf{n l}}(\mathbf{r})$, where $\mathbf{J}_{\mathbf{n l}}(\mathbf{r})$ is the current density from the contribution of nonlocal potential and can be obtained by solving a Poisson equation. For details see C. S. Li, L. H. Wan, Y. D. Wei, and J. Wang, Nanotechnology 19, 155401 (2008). 